\title{
Gap anisotropy and universal pairing scale in a spin-fluctuation model of cuprate superconductors
}

\author{
Ar. Abanov, ${ }^{1}$ A. V. Chubukov, ${ }^{2}$ and M. R. Norman ${ }^{3}$ \\ ${ }^{1}$ Department of Physics, Texas A\&M University, College Station, Texas 77843, USA \\ ${ }^{2}$ Department of Physics, University of Wisconsin, Madison, Wisconsin 53706, USA \\ ${ }^{3}$ Materials Science Division, Argonne National Laboratory, Argonne, Illinois 60439, USA
}

(Received 23 October 2008; published 19 December 2008)

\begin{abstract}
We consider the evolution of $d_{x^{2}-y^{2}}$ pairing, mediated by nearly critical spin fluctuations, with the coupling strength. We show that the onset temperature for pairing, $T^{*}$, smoothly evolves between weak and strong couplings passing through a broad maximum at intermediate coupling. At strong coupling, $T^{*}$ is of order of the magnetic exchange energy $J$. We argue that for all couplings, pairing is confined to the vicinity of the Fermi surface. We also find that thermal spin fluctuations only modestly reduce $T^{*}$, even at criticality, but they substantially smooth the gap anisotropy. The latter evolves with coupling being the largest at weak coupling.
\end{abstract}

DOI: 10.1103/PhysRevB.78.220507

PACS number(s): 74.20.Mn, 74.25.Jb, 74.72.-h

\section{INTRODUCTION}

Understanding the origin of the pseudogap in high- $T_{c}$ cuprate superconductors is a key problem. ${ }^{1}$ Some argue that the pseudogap originates from (quasi-)long-range order in a nonpairing channel (two-gap scenario). ${ }^{2}$ Others argue instead that the pseudogap is a phase in which fermions already form singlet pairs, but long-range superconducting coherence is not yet established (one-gap scenario). . $^{3,4}$

The theories within the one-gap scenario can be broadly separated into two classes_- "strong-coupling" theories which consider a doped Mott insulator and "weak-coupling" theories which assume a normal metallic state at large dopings. It is widely accepted that the cuprates display a crossover from Mott-type behavior in the underdoped regime to Fermi-liquid-like behavior in the overdoped regime with the superconducting dome straddling these two regimes. Quantitatively, strong and weak couplings are the limits of small and large values of the dimensionless coupling $u$, which scales as $U / W$, where $U$ is the effective Hubbard interaction and $W$ is the bandwidth. For $u<1$, it is natural to assume that pairing is confined to near the Fermi surface and can be thought of as mediated by a bosonic "glue," the most natural candidate being collective excitations in the spin channel, enhanced around $\mathbf{Q}=(\pi / a, \pi / a)$ (i.e., antiferromagnetic spin fluctuations). For $u>1$, it has been $\operatorname{argued}^{5}$ that the notion of a bosonic glue is meaningless since the dynamics of the superexchange interaction, $J$, occurs only for energy scales of order $U$, but this has been challenged based on simulations of the Hubbard model, ${ }^{6}$ which found that pairing originates mostly from energies of order $J$.

In this Rapid Communication, we argue that the nature of the pairing is similar for both small and large $u$. We show that the onset temperature for pairing, which is the pseudogap $T^{*}$ in a one-gap scenario, smoothly evolves between weak and strong couplings, passing through a broad maximum at intermediate coupling, where $T^{*}$ is a fraction of the Fermi energy (Fig. 1). At large $u, T^{*} \sim J$. Still, we find that even in this limit, pairing is confined to the vicinity of the Fermi surface. The only real difference between weak and strong couplings is the range of the Fermi-surface momenta involved in pairing-for $u<1$, pairing comes from regions around the hot spots (Fermi momenta connected by $Q)$, while for $u>1$ the whole Fermi surface is involved in pairing. We show that $T^{*}$ weakly depends on the magnetic correlation length $\xi$ and can easily reach $300-500 \mathrm{~K}$ for $u$ $\sim O(1)$

We also discuss the special role of static thermal fluctuations, which scatter with zero energy transfer and therefore act as nonmagnetic impurities that are pairbreaking for $d_{x^{2}-y^{2}}$ symmetry. ${ }^{7,8}$ Static fluctuations are particularly relevant for $\xi=\infty$ when their contributions to the mass renormalization and the pairing vertex diverge. We found that static thermal fluctuations do reduce $T^{*}$ somewhat, but $T^{*}$ still remains finite for $\xi=\infty$.

Finally, we discuss the anisotropy of the $d_{x^{2}-y^{2}}$ gap. We find that for small $u$, the gap is anisotropic and drops in magnitude upon deviation from the hot spots. At large $u$, the gap is close to the $\cos \left(k_{x} a\right)-\cos \left(k_{y} a\right)$ form.

\section{MODEL}

We consider an approach to pairing from the Fermi-liquid region of large dopings. We assume that the strongest fermion-fermion interaction is in the spin channel for momentum transfers near $Q$. The low-energy physics of such a Fermi liquid is captured by a semiphenomenological spinfermion model, which reduces the interaction between lowenergy fermions to the exchange of two-particle collective modes in the spin channel. ${ }^{9}$ The model describes fermions interacting via an effective four-fermion vertex $\Gamma_{q}(\Omega)$ $=(U / 2) D_{q}(\Omega)$, where $U$ is the irreducible Hubbard interaction and $D_{q}(\Omega)$ is the dynamic spin susceptibility. The phenomenological input is the static $D_{q}(0)$, which comes from fermions with high energies of the order of the bandwidth. The frequency dependence of $D_{q}(\Omega)$ comes from low-energy fermions and is computed within the model. In earlier work, $D_{q}(0)$ was assumed to have an Ornstein-Zernike (OZ) form $D_{q}^{-1}(0)=\xi^{-2}+|\mathbf{q}-\mathbf{Q}|^{2}$, where $\xi$ is the magnetic correlation length. We will use this form of $D_{q}(0)$, and also a related form $D_{q}^{-1}(0)=\xi^{-2}+|\mathbf{q}-\mathbf{Q}|^{2}+b\left[\left(q_{x}-\pi / a\right)^{4}+\left(q_{y}-\pi / a\right)^{4}\right]$ with $b>0$, to model inelastic neutron-scattering experiments that show that the spin fluctuations decrease faster with deviation from $Q$ than the $\mathrm{OZ}$ form predicts. ${ }^{10}$ 


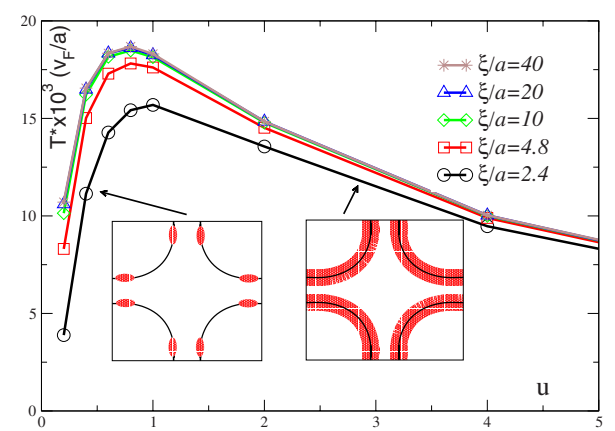

FIG. 1. (Color online) The onset temperature $T^{*}$ for $d_{x^{2}-y^{2}}$ pairing vs the dimensionless coupling $u$ for an Ornstein-Zernike form for the static spin propagator near $Q$ and various values of the magnetic correlation length $\xi$ with $v_{F} / a=1 \mathrm{eV}$. The shading in the insets represents those parts of the Brillouin zone where pairing is confined to. For both small and large $u$, pairing is confined to the vicinity of the Fermi surface.

The effective four-fermion vertex gives rise to fermion and boson self-energies and to an attractive pairing interaction in the $d_{x^{2}-y^{2}}$ channel. ${ }^{11}$ We will adopt Eliashberg theory which has been justified earlier on the basis that collective bosons are slow compared to fermions. ${ }^{9,12}$ In this theory, the boson self-energy reduces to the Landau damping term

$$
D_{q}^{-1}(m)=D_{q}^{-1}(0)+\frac{2 \pi T|m|}{\Gamma}, \quad \Gamma=\frac{3 a}{16} \frac{v_{F}}{u},
$$

where $\Omega=2 \pi T m$ and $u=3 U a /\left(8 \pi v_{F}\right)$ is the dimensionless coupling with $v_{F}$ the nodal velocity. The linearized gap equation has the form

$$
\begin{aligned}
\Delta_{k}(n)= & -\frac{u a}{\pi} \sum_{m} \oint d k^{\prime} \nu_{k^{\prime}} \frac{\Delta_{k^{\prime}}(m)+\Delta_{k}(n) \frac{2 m+1}{2 n+1}}{|2 m+1|} \\
& \times \frac{1}{(\xi / a)^{-2}+a^{2}\left|\mathbf{k}-\mathbf{k}^{\prime}-\mathbf{Q}\right|^{2}+2 \pi|n-m| \frac{a^{2} T}{\Gamma}}
\end{aligned}
$$

where $\omega=\pi T(2 m+1)$, the integration over $k^{\prime}$ is along the Fermi surface, and $\nu_{k}$ is the density of states normalized such that $\nu=1$ along the nodal direction. We emphasize that the boson self-energy and the pairing come from the same interaction and $\Gamma$ contains the same dimensionless coupling $u$ as the pairing kernel.

The temperature only appears explicitly in Eq. (2) in the Landau damping term; hence $T^{*} \propto \Gamma / a^{2}$, where the prefactor depends on $u$ and $\xi$. Using the definition of $\Gamma$, we then obtain

$$
T^{*}=\frac{v_{F}}{a} f_{\xi}(u),
$$

where $f_{\xi}(u)$, a function of $u$ and $\xi$, is evaluated below.

We also consider the fermionic self-energy $\Sigma_{K}(\omega)$ $=\Sigma_{k}(n)$ and the mass renormalization $Z_{k}(n)=1$ $+\Sigma_{k}(m) /(\pi T(2 m+1))$. The latter effectively measures the coupling strength along the Fermi surface and is given by

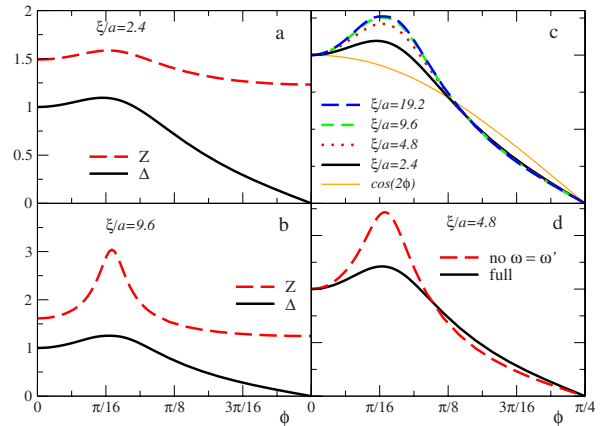

FIG. 2. (Color online) The mass renormalization $Z_{k}(0)$ and the pairing gap $\Delta_{k}(0)$ for small $u=0.2$. Panels (a) and (b) show the results for two different $\xi$; panel (c) shows the angular dependence of the gap compared to the $\cos \left(k_{x} a\right)-\cos \left(k_{y} a\right)$ form; panel (d) shows $\Delta_{k}(0)$ for $\xi=4.8 a$ with and without the pairbreaking contribution from the static spin fluctuations [the term with $n=m$ in Eq. (2)]. Without the static contribution, $Z_{k}(0) \equiv 1$.

$Z_{k}(n)=1+\frac{u}{a \pi} \sum_{m} \oint d k^{\prime} \nu_{k^{\prime}} \frac{\operatorname{sgn}(2 m+1)}{2 n+1} D_{k-k^{\prime}}(|n-m|)$.

One can easily verify that $Z_{k}(0)=1$ $+(u /(a \pi)) \oint d k^{\prime} \nu_{k^{\prime}} D_{k-k^{\prime}}(0)$ is independent of the Landau damping. For large $u, Z \gg 1$ along the entire Fermi surface; for small $u$, but large $\xi$, it is still large near the hot spots $k$ $=k_{h}$, where $Z_{k_{h}}(0)=1+u(\xi / a)$. We verified that for $\xi \gg a$, fermions relevant for pairing have $Z_{k}>1$, i.e., pairing in the critical regime $\xi \gg 1$ is a strong-coupling phenomenon for all $u$.

\section{RESULTS}

The results of the numerical calculations for $T^{*}(u), \Delta_{k}(n)$, and $Z_{k}(n)$ for the OZ form of $D_{q}(0)$ are presented in Figs. 1-3 (we used a $t-t^{\prime}$ model for the dispersion and 142 Matsubara frequencies). $\Delta_{k}(n)$ monotonically decreases with increasing frequency (not shown). The dependences of $\Delta_{k}(n)$ and $Z_{k}(n)$ for $k$ along the Fermi surface are similar for different $n$, so we only present the results for the lowest $n=0$ $(\omega=\pi T)$.

In Fig. 1 we show the dependence of $T^{*}$ on the dimensionless coupling $u$. We see that $T^{*}$ initially increases with $u$, passes through a broad maximum at $u \sim 1$, and then decreases eventually as $1 / u$.

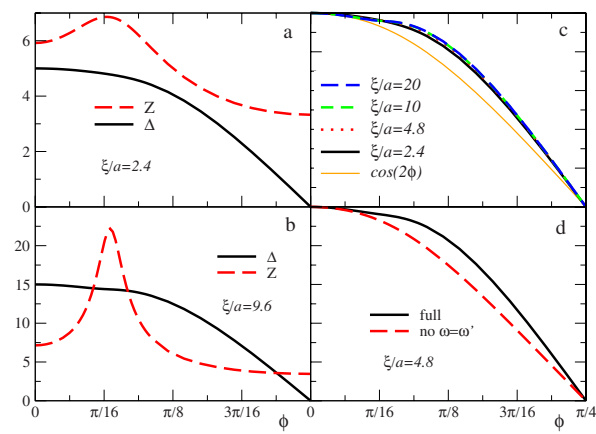

FIG. 3. (Color online) Same as Fig. 2 but for large $u=2$. 
In Fig. 2 we show the results for $Z_{k}(0)$ and $\Delta_{k}(0)$ for small $u=0.2$. We see that $Z_{k}(0)$ is enhanced near the hot spots and then drops to near its noninteracting value $(Z=1)$, which implies that only the hot regions are relevant for pairing. $\Delta_{k}(0)$ also has a maximum at the hot spots, leading to a significant deviation from the $\cos \left(k_{x} a\right)-\cos \left(k_{y} a\right)$ form. Moreover, the slope of $\Delta_{k}(0)$ near the node is smaller than this form.

In Fig. 3 we show the results for large $u=2$. We see that $Z$ is enhanced along the entire Fermi surface. The deviation of the gap anisotropy from the $\cos \left(k_{x} a\right)-\cos \left(k_{y} a\right)$ form is much weaker than at small $u$, and the slope of $\Delta_{k}(0)$ near the node is larger than this form.

In Fig. 4 we show how $T^{*}(u)$ changes with the deviation of the static susceptibility from the OZ form. We see that the magnitude of $T^{*}(u)$ increases for $b>0$, i.e., when $D_{q}(0)$ drops faster from $Q$ than the $\mathrm{OZ}$ form.

\section{ANALYTICAL REASONING}

All these results can be understood analytically. Consider first why $T^{*}$ remains finite when $\xi$ diverges despite the fact that thermal spin fluctuations are pairbreaking for a $d$-wave gap. We recall that Eliashberg theory is a set of two coupled equations for the pairing vertex $\Phi(n)$ and self-energy $\Sigma(n)$. They reduce to independent equations for $\Delta(n)$ and $Z(n)$ by exploiting the definitions $\Phi(n)=\Delta(n) Z(n)$ and $\Sigma(n)=\pi T(2 n$ $+1)(Z(n)-1)$. The static thermal contribution to Eq. (2) (the term with $n=m$ ) has the form

$$
-\frac{u a}{\pi|2 n+1|} \oint d k^{\prime} \nu_{k^{\prime}} \frac{\Delta_{k^{\prime}}(n)+\Delta_{k}(n)}{(\xi / a)^{-2}+a^{2}\left|\mathbf{k}-\mathbf{k}^{\prime}-\mathbf{Q}\right|^{2}},
$$

where the terms with $\Delta_{k}$ and $\Delta_{k^{\prime}}$ are the contributions from $\Sigma$ and $\Phi$, respectively. Each diverges at $\xi=\infty$, and taken alone, would drive $T^{*}$ to zero. However, the sum of the two remains finite because by symmetry $\Delta_{k+Q}=-\Delta_{k}$ and $T^{*}$ does not vanish at $\xi=\infty$. Note that there is no such cancellation for $Z_{k}(n)$, which diverges at a hot spot when $\xi=\infty$.

Consider next the dependence of $T^{*}$ on $u$ for $\xi=\infty$. At small $u$, pairing is confined to hot regions $\left(k \approx k_{h}\right)$, and the momentum dependence of the static $D_{k-k^{\prime}}$ can be approximated by $\left|\mathbf{k}_{h}-\mathbf{k}^{\prime}\right|^{2}$. The integral over $k^{\prime}$ in Eq. (2) can then be evaluated analytically, and Eq. (2) reduces to a onedimensional integral equation for $\Delta_{k_{h}}(n)$. Simple power counting then yields $T^{*}=B\left(\Gamma / a^{2}\right) u^{2}$, where $B$ is a constant. A numerical solution gives ${ }^{13} T^{*} \approx 0.68\left(\Gamma / a^{2}\right) u^{2}=0.13\left(v_{F} / a\right) u$, i.e., $f_{\infty}(u \ll 1) \approx 0.13 u$.

The reduction in $T^{*}(u)$ at large $u$ is peculiar to $d$-wave pairing. The argument is that, as $u$ increases, $T^{*}$ initially also increases, and at some $u$ the dynamic term in $D_{k-k^{\prime}}(n-m)$ becomes comparable to a typical $\left|\mathbf{k}-\mathbf{k}^{\prime}-\mathbf{Q}\right|^{2}$ term along the Fermi surface, which determines the attractive $d$-wave component of the static interaction. A further increase in $T$ would make the effective interaction less momentum dependent and hence would reduce the $d$-wave attraction. The balance is reached when $T^{*} / \Gamma$ is a constant, i.e., when $f_{\infty}(u) \propto 1 / u$. Numerically, $f_{\infty}(u) \sim 0.056 / u$ for the OZ form of $D_{q}(0)$. This can be re-expressed as $T^{*} \sim 0.47\left(v_{F} / a\right)^{2} / U \sim 0.5 J$, where $J$ $\approx\left(v_{F} / a\right)^{2} / U$ is the exchange integral of the underlying

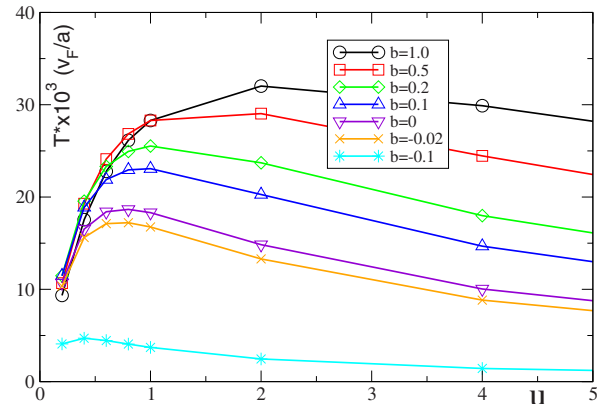

FIG. 4. (Color online) The onset temperature $T^{*}(u)$ for different forms of the static susceptibility $D_{q}(0)$ (see text). The magnitude of $T^{*}(u)$ increases as $D_{q}(0)$ drops faster with deviation from $Q$.

Heisenberg model at half filling. ${ }^{14}$ We interpret this as evidence that pairing of incoherent fermions in the spin-fermion model and the creation of singlet pairs upon doping a MottHeisenberg insulator describe the same physics from different perspectives.

This conclusion is only valid, though, if the pairing remains confined to the vicinity of the Fermi surface. To verify this, from the integral over $\epsilon_{k}$ which leads to Eq. (2), we can estimate a typical $\left|\mathbf{k}-\mathbf{k}_{F}\right|$ transverse to the Fermi surface from $v_{F}\left|\mathbf{k}-\mathbf{k}_{F}\right| \sim \pi T^{*}(2 n+1) Z(n)$, with typical $n$ of order 1 for all $u$. For $Z(n)$ we use the quantum critical, $\xi=\infty$, form ${ }^{9}$ $Z(n) \approx 2 a^{-1} u \sqrt{\Gamma /\left(\pi T^{*}(2 n+1)\right)}$. Substituting it, we find that for large $u,\left|\mathbf{k}-\mathbf{k}_{F}\right| \sim(3 / 8) \sqrt{\pi T^{*} / \Gamma} \sim 0.1(\pi / a)$. This $\left|\mathbf{k}-\mathbf{k}_{F}\right|$ is numerically much smaller than $k_{F} \sim 0.8(\pi / a)$, i.e., even for large $u$ pairing involves fermions from a narrow shell around the Fermi surface.

Consider next the variation of the gap along the Fermi surface. For small $u$, the anisotropy of the gap is a consequence of the fact that the pairing problem is confined to a region near the hot spots with width $\delta k \sim k_{F} u$. At strong coupling, the nonconfinement of $\Delta_{k}$ to hot spots is due to a cancellation between the anisotropies of $\Phi$ and $Z$ when dividing to form $\Delta$. The extent to which the residual nonsingular pairbreaking contribution from thermal spin fluctuations affects the shape of the gap can be verified numerically. In the last panels in Figs. 2 and 3, we show the gap variation along the Fermi surface with and without the pairbreaking contribution to the gap equation. We see that $\Delta_{k}$ near the node becomes larger than the $\cos \left(k_{x} a\right)-\cos \left(k_{y} a\right)$ form when the pairbreaking contribution is included.

Finally, we consider the variation of the overall scale for $T^{*}$ with $b$ (Fig. 4). To understand this, we recall that for the OZ form of $D_{q}(0)$, a strong reduction in $T^{*}$ compared to the asymptotic small $u$ form $T^{*} \approx 0.13\left(v_{F} / a\right) u$ was due to the interplay between the $2 \pi T^{*}|n-m| / \Gamma$ term and the maximum $\left|\mathbf{k}-\mathbf{k}^{\prime}\right|^{2}$ along the Fermi surface. Once $D_{q}(0)$ becomes steeper with deviation from $Q(b>0)$, the typical $k$ and $k^{\prime}$ get closer to the hot spots, the restriction on $\left|\mathbf{k}-\mathbf{k}^{\prime}\right|$ becomes less relevant, and $T^{*}$ increases. For $b<0$, the situation is the opposite, geometrical restrictions become more relevant, and $T^{*}$ rapidly decreases.

\section{COMPARISON WITH EARLIER STUDIES}

Several groups did extensive studies of $T^{*}$ within the Eliashberg theory for the spin-fermion model. ${ }^{12,15}$ They 
treated $\Gamma$ as an independent parameter, i.e., they did not express it in terms of $u$. Our results agree with these studies if we use the same $v_{F}, u$, and $\Gamma$ as they did. Monthoux and Pines ${ }^{11}$ studied $T^{*}(u)$ at small $u$ and noticed that its slope decreases as the coupling increases, which is consistent with Fig. 1. Schuttler and Norman ${ }^{15}$ found a saturation of $T^{*}$ when measured in units of $\Gamma$, but for a model in which $D(q, \Omega)$ had the factorized form $D_{1}(q) D_{2}(\Omega)$, and a strong $T$ dependence of $\Gamma$ is introduced phenomenologically. Within the spinfermion model, though, the $T$ dependence of $\Gamma$ is weak. Our results also agree with FLEX calculations for the Hubbard model. ${ }^{16-18}$ In these calculations, $\Gamma$ is obtained selfconsistently, as in our theory. For $U=4 t$ and $v_{F} / a \approx 2 t$ $(u \approx 0.25)$, our $T^{*} \approx 0.02 t$ is in good agreement with Refs. 16-18. This value is also in good agreement with twoparticle self-consistent calculations, ${ }^{19}$ dynamical cluster approximation, ${ }^{20}$ and cluster DMFT, ${ }^{21}$ which also yield that the $d$-wave order parameter scales as $J$ at large $u .^{22}$

\section{COMPARISON WITH THE CUPRATES}

Both $\xi$ and $u$ increase with underdoping, leading to an initial increase and eventual saturation of $T^{*}$. To find the value of $T^{*}$ at saturation, we use $v_{F} / a \sim 1 \mathrm{eV}$, noting that $v_{F}$ is the "bare" velocity as obtained in band theory. For the OZ form of the static $D_{q}(0)$, we obtain $T^{*} \sim 0.02 v_{F} / a$ $\sim 200-250 \mathrm{~K}$ for $u \sim O(1)$. For $b=1$, this temperature increases to over $350 \mathrm{~K}$ (Fig. 4) and becomes even larger for larger $b$. This shows that a spin-mediated pairing interaction is strong enough to account for experimental values of $T^{*}$ even in the underdoped regime. We emphasize that the maximum $T^{*}$ depends weakly on $u$ for $u \sim O(1)$; hence, a precise value of $u$ is not necessary for an estimate of $T^{*}$.

In addition, for optimal and underdoped cuprates, we find a gap close to the $\cos \left(k_{x} a\right)-\cos \left(k_{y} a\right)$ form. This is consistent with photoemission ${ }^{23}$ below $T_{c}$ and with the scenario that the Fermi arcs above $T_{c}$ appear because of thermal broadening of the spectral function. ${ }^{4}$

\section{CONCLUSIONS}

In this Rapid Communication, we analyzed how $d_{x^{2}-y^{2}}$ pairing mediated by nearly critical spin fluctuations varies with the coupling strength. We argued that the onset temperature for pairing $T^{*} \approx\left(v_{F} / a\right) f_{\xi}(u)$ smoothly evolves between weak and strong couplings, passing through a shallow maximum at $u \sim 1$. At large $u, T^{*} \sim v_{F} / u \sim J$. For all $u$, pairing is confined to the vicinity of the Fermi surface. We also argued that singular pairbreaking contributions from static spin fluctuations cancel out in the gap equation, while the residual pairbreaking terms only modestly reduce $T^{*}$ and at the same time smooth the angular dependence of the gap.

As Mott physics is certainly present near half filling, where $T^{*}$ reaches its largest values, our $T^{*}(u)$ should only be taken as an estimate. Still, the fact that $T^{*}$ is in the experimental range is in support of a one-gap scenario in which the instability at $T^{*}$ occurs in the particle-particle channel due to interactions with spin fluctuations.

\section{ACKNOWLEDGMENTS}

We acknowledge helpful discussions with M. Eschrig, I. Eremin, A. J. Millis, A.-M. Tremblay, and I. Vekhter, support from the Welch Foundation (Ar.A.), NSF under Grant No. DMR 0604406 (A.V.C.), and the Office of Science, U.S. DOE under Contract No. DE-AC02-06CH11357 (M.R.N.).
${ }^{1}$ M. R. Norman et al., Adv. Phys. 54, 715 (2005).

${ }^{2}$ A. Perali et al., Phys. Rev. B 62, R9295 (2000); S. Chakravarty et al., ibid. 63, 094503 (2001); B. Kyung et al., ibid. 73, 165114 (2006); C. M. Varma and L. Zhu, Phys. Rev. Lett. 98, 177004 (2007).

${ }^{3}$ P. W. Anderson et al., J. Phys.: Condens. Matter 16, R755 (2004); N. Trivedi and M. Randeria, Phys. Rev. Lett. 75, 312 (1995); V. Emery and S. Kivelson, Nature (London) 374, 434 (1995); M. Franz and A. J. Millis, Phys. Rev. B 58, 14572 (1998); M. R. Norman et al., ibid. 57, R11093 (1998).

${ }^{4}$ M. R. Norman et al., Phys. Rev. B 76, 174501 (2007); A. V. Chubukov et al., ibid. 76, 180501(R) (2007).

${ }^{5}$ P. W. Anderson, Science 317, 1705 (2007).

${ }^{6}$ T. A. Maier et al., Phys. Rev. Lett. 100, 237001 (2008); A.-M. S. Tremblay (private communication).

${ }^{7}$ A. A. Abrikosov and L. P. Gor'kov, Zh. Eksp. Teor. Fiz. 12, 1243 (1961).

${ }^{8}$ A. J. Millis et al., Phys. Rev. B 37, 4975 (1988).

${ }^{9}$ See, e.g., Ar. Abanov et al., Adv. Phys. 52, 119 (2003), and references therein.

${ }^{10}$ S. Pailhes et al., Phys. Rev. B 71, 220507(R) (2005).
${ }^{11}$ D. J. Scalapino, Phys. Rep. 250, 329 (1995); P. Monthoux and D. Pines, Phys. Rev. B 49, 4261 (1994).

12 A. J. Millis, Phys. Rev. B 45, 13047 (1992).

${ }^{13}$ Ar. Abanov et al., Europhys. Lett. 54, 488 (2001).

${ }^{14}$ For a $t-t^{\prime}$ model with $t^{\prime} \sim-0.3 t$ and a nodal $\mathbf{k}_{F}$ $=(0.4 \pi / a, 0.4 \pi / a)$, the holelike Fermi surface is nearly circular around $(\pi, \pi)$, with $v_{F} / a \approx 2 t$.

${ }^{15}$ H.-B. Schuttler and M. R. Norman, Phys. Rev. B 54, 13295 (1996).

${ }^{16}$ P. Monthoux and D. J. Scalapino, Phys. Rev. Lett. 72, 1874 (1994).

${ }^{17}$ T. Dahm and L. Tewordt, Phys. Rev. B 52, 1297 (1995); D. Manske et al., ibid. 67, 134520 (2003).

${ }^{18}$ St. Lenck et al., Phys. Rev. B 50, 10149 (1994).

${ }^{19}$ B. Kyung et al., Phys. Rev. B 68, 174502 (2003).

${ }^{20}$ T. A. Maier et al., Phys. Rev. Lett. 95, 237001 (2005).

${ }^{21}$ K. Haule and G. Kotliar, Phys. Rev. B 76, 104509 (2007).

${ }^{22}$ S. S. Kancharla et al., Phys. Rev. B 77, 184516 (2008); D. Senechal and A.-M. S. Tremblay, Phys. Rev. Lett. 92, 126401 (2004).

${ }^{23}$ A. Kanigel et al., Phys. Rev. Lett. 99, 157001 (2007). 